\title{
Comparing Acute Aerobic vs. Resistance Exercise (CAARE) in young and older adults: Design and methods of a randomized acute exercise trial on the immune system
}

Melissa M Markofski ( $\square$ mmarkofs@central.uh.edu )

London Metropolitan University Libraries https://orcid.org/0000-0003-0214-4246

Rachel M Graff

University of Houston

Kristofer Jennings

University of Texas MD Anderson Cancer Center

Study protocol

Keywords: inflammation, physical fitness, immune

Posted Date: March 4th, 2020

DOI: https://doi.org/10.21203/rs.3.rs-15250/v1

License: (c) (i) This work is licensed under a Creative Commons Attribution 4.0 International License.

Read Full License 


\section{Abstract}

Background: It is well accepted that exercise has numerous health benefits, including reducing the risk of developing cardiometabolic diseases and supporting a healthy immune system. Although these benefits are widely recognized, variations in the exercise response still exist. The aim of this study is to explore the variations that may be partially explained by participant age, physical activity status, and/or mode of exercise.

Methods: The CAARE (Comparing Acute Aerobic vs. Resistance Exercise) study is a randomized crossover design trial. Participants are young (18-30 years) or older (55-75 years), and within each age group are either physically active or inactive. All participants complete two acute exercise sessions of similar intensity and duration: a resistance training bout and a treadmill exercise bout. Blood is collected before exercise (PRE), immediately after exercise (POST), and after one hour of recovery (RECOV). Fresh blood is used for flow cytometry analyses to phenotype immune cells. Serum is stored for future analyses. The primary outcome variables are monocyte and T-cell phenotype.

Discussion: CAARE is the first study to compare the immune cell response to acute exercise when including age, physical activity status, and mode of exercise.

Clinical trial registration: This research study was registered at clinicaltrials.gov NCT03794050 in January 4, 2019. https://clinicaltrials.gov/ct2/show/NCT03794050

\section{Background}

It is well accepted that regular physical exercise has many health benefits, including the reduced risk of developing cardiometabolic diseases such as cardiovascular disease and type 2 diabetes. The risk of developing these diseases increases with age, leading many to state that these diseases are age-related. However, with increasing age is also a decrease in physical activity. This implies that many of the diseases typically linked to age, such as cardiovascular disease, may instead be related to an ageassociated decline in physical activity. Further complicating this relationship is strong evidence that both physical inactivity and aging cause changes in the immune system-specifically, increases in inflammation and senescent cells.

One source of inflammation is pro-inflammatory monocytes (CD14+CD16+). Monocytes can be of a "classical" phenotype (CD14+CD16-), or express CD16 and be a pro-inflammatory phenotype that produces a high number of pro-inflammatory cytokines(1). Inactive but otherwise healthy adults have a significantly higher percentage of circulating "inflammatory" monocytes (CD14+CD16+) than agematched physically active controls $(2,3)$. This is significant, because there is evidence that the inflammatory CD14+CD16+ monocytes are elevated in people with cardiovascular disease(4). Fortunately, the phenotype can be influenced by lifestyle-specifically, exercise and physical activity. Even though physically inactive older adults had a higher percentage of circulating inflammatory monocytes, 
when the inactive subjects completed a supervised exercise training program, the inflammatory monocytes were reduced to the same level as the physically active controls(3).

Similarly, aging has been associated with an accumulation of highly-differentiated CD8+ T-cells, which often occurs with a concurrent reduction in the naïve T-cell repertoire. These changes are associated with impaired immunity and increases in chronic systemic inflammation(5). However, there is evidence to support our hypothesis that physical inactivity, rather than aging, is a greater predictor of these negative T-cell changes. Indeed, Spielmann et al.(6) reported that cardiorespiratory fitness level was a greater predictor of T-cell senescence than age. This finding supports the benefit of maintaining physical activity throughout the lifespan to possibly counteract the negative T-cell changes that are often attributed to aging.

Although it is known that CD14+CD16+ monocytes and highly-differentiated CD8+ T-cells mobilize in response to an acute exercise bout(7) and that they are influenced by regular training $(2,3)$, little is known about the potential differences in response due to age, physical activity (training) status, and mode of exercise. Therefore, the purpose of this study is to examine the acute exercise-induced response when these three factors (age, physical activity status, mode) are included in the experimental design. The primary variables are the phenotypes of monocytes and $\mathrm{T}$ cells. We hypothesize that physical activity status will have a bigger influence than age on our primary variables.

\section{Methods}

Research primary objective and design

The primary objective of the CAARE study is to determine the differences in the immune response to an acute bout of exercise when considering age and physical activity status of the individual and mode of exercise. CAARE is a randomized, complete cross-over $2 \times 2 \times 2$ (age $\times$ physical activity status $\times$ mode) factorial design with three timepoints (pre-exercise, post-exercise, and recovery). Apparently healthy young (18-30 years) and older (55-75 years) adults who are currently meeting ACSM exercise recommendations(8) (physically active) or are not exercising (physically inactive) will participate in two moderate to vigorous exercise bouts of different modes: a stacked weight machine resistance exercise bout (RE) and a motor-driven treadmill cardiorespiratory exercise (CRE) bout. Participants are categorized as young/old and active/inactive, then stratified by sex and randomized to which exercise bout they will complete first. We hypothesize that physical activity status will have a bigger influence than age on our variables.

Participant eligibility and recruitment

We are recruiting healthy adults for this study. Participants are recruited from flyers, attendance at health or community fairs, classrooms (on campus and at community centers), and word-of-mouth. To be 
eligible for participation, potential participants must be classified as "young" and be 18-30 years of age, or "older" and 55-75 years. Females in the "older" age classification must be post-menopausal. Within each age classification, we are recruiting participants who are physically active or inactive. Physically active is defined by the American College of Sports Medicine (ACSM) and American Heart Association primary physical activity recommendations(8) of at least 150 minutes per week of moderate intensity cardiorespiratory exercise training, 60 minutes per week of vigorous intensity cardiorespiratory exercise training, or some combination of moderate and vigorous cardiorespiratory exercise training. In addition to the cardiorespiratory exercise training, the physically active exercise participants must participate in at least two days per week of skeletal muscle strength and endurance training exercises. Physically inactive participants complete less than two days per week of moderate intensity cardiorespiratory exercise training of less than 60 minutes weekly total, no vigorous intensity cardiorespiratory exercise training, and no skeletal muscle strength and endurance training exercises.

Participant exclusion criteria includes: contraindications to moderate or vigorous exercise; recent illness; instructions from a healthcare provider to not exercise; range of motion limitations that would prevent proper exercise form; use of prescription or over the counter medications known to influence immune function (i.e. daily NSAID, statins, bisphosphonates, steroids, etc.); report cardiovascular, respiratory, metabolic, or renal disease, with the exception of controlled hypertension (as defined by resting BP below 140/90); self-reported uncontrolled asthma; gravid; excessive travel that would impact study visit schedule; $>30 \mathrm{BMI}<18.5 \mathrm{~kg} \cdot \mathrm{m}^{-2}$; or unable to refrain from alcohol or recreational drugs for $24 \mathrm{~h}$ prior to study visits. This study was approved by the institutional review board at the University of Houston (STUDY00001191) and the study setting is the Laboratory of Integrative Physiology In the Department of Health and Human Performance at the University of Houston (academic research laboratory).

Participant enrollment and data collection

After a potential participant expresses interest in the study, they are provided with information to help them determine if they meet self-reported requirements for participation (i.e. a list of exclusionary medications, been instructed by a health care member to not exercise, known cardiometabolic diseases, etc.). If the participant felt they met the basic inclusion/exclusion criteria, they were scheduled for study visit one at the Laboratory of Integrative Physiology (Figure 1). After written informed consent was obtained by a co-investigator, the following measures were obtained: height, weight, blood pressure, body composition, medical history, physical activity frequency, Positive and Negative Affect Schedule (PANAS) and Profile of Mood States Questionnaire (PMSQ), and heart rate variability (HRV). If the participant met all the study qualifications, they were scheduled for visit two. Visit two occurred immediately after visit one or up to fourteen days later and consists of an estimated treadmill $\mathrm{O}_{2}$ peak test, strength tests, body composition estimation, and a resistance exercise familiarization session. 
Visits three and four are the primary data collection visits. In a random counterbalanced order, participants complete a CRE bout at one visit and a RE bout at the other visit. Participants come to the laboratory after an overnight fast (water ad libitum was allowed and encouraged). After resting in a seated position for 15 minutes, a pre-exercise (PRE) blood sample was collected. The participant completes a five-minute warm-up on a motor-driven treadmill, then performs their assigned exercise bout for the visit. The cardiorespiratory bout consists of 30 minutes of walking or running at $70 \%$ of heart rate reserve (HRR). The resistance training bout consists of three sets of eight exercises at $70 \% 1 \mathrm{RM}$, and takes approximately 30 minutes to complete.

\section{Measurements and assessments}

Physical activity assessment and monitoring

After consent, participants are asked to complete an exercise frequency questionnaire. This questionnaire lists commonly performed exercise and physical activities. The participant is asked to estimate pace (if applicable), days per week spent doing the activity, and time per day spent doing the activity. Accelerometers (ActiGraph WGT3X-BT, ActiGraph LLC, Pensacola, FL) are used to objectively measure physical activity. After enrollment, participants are asked to wear the accelerometer on their ankle for one week. The accelerometry data is used to confirm the self-reported physical activity level in the exercise frequency questionnaire.

Body composition

Body composition estimations are collected with a whole-body dual energy $\mathrm{x}$-ray absorptiometry (Horizon A, Hologic, Marlborough, MA USA). Participants are scanned in the morning after an overnight fast and laying on the table for five minutes.

Cardiorespiratory fitness

A two-stage motorized sub-maximal treadmill test is used to estimate $0_{2}$ peak. Each stage is three minutes long, and heart rate is noted in the last 15 seconds of each stage. The participants are asked to walk or run both stages (their preference). The incline is increased for the second stage, and if needed to increase the heart rate $>20$ BPM the speed is also increased. The $0_{2}$ is calculated for each stage, and the slope of the line is calculated and used to estimate $0_{2}$ peak.

Skeletal muscle strength 
Participants perform an eight-repetition maximum test on each of the eight pieces of equipment used for the resistance exercise study visit. The one repetition maximum (1RM) is estimated from the eight repetition weight(9) , and used to calculate the weight loads on the resistance exercise study visit.

Cardiorespiratory endurance exercise bout

The heart rate reserve (HRR) method is used to calculate CRE exercise study visit intensity. Specifically, the CRE bout is 30 minutes of walking at $60 \%-70 \%$ HRR on a motor-driven treadmill. The 30 -minute bout is preceded by five minutes and followed by two minutes of treadmill walking at a pace self-selected by the study participant. This two-minute cool-down occurs after the POST blood sample is collected from the participant. During the walking bout, HR is monitored to ensure the bout is within the target HR zone (60\%-70\% HRR). RPE is collected using the 6-20 Borg Scale.

Resistance exercise bout

The RE bout is approximately 30 minutes at $70 \%$ of estimated $1 \mathrm{RM}$ on eight stacked-weight machines (leg press, leg extension, leg curl, weighted calf raise, chest press, lateral pull down, seated row, and triceps extension). Participants perform three sets of 8- 12 repetitions, with an approximate 30 seconds of rest between sets. All sets at one station are completed before the participant moves to the next station. As with the CRE bout, the bout is preceded by a five minute warm-up walk on the treadmill, and followed by a two minute cool-down walk. Both paces are self-selected by the participant, and the POST blood sample is collected prior to the cool-down.

Biochemical assessments

Blood samples are collected pre-exercise (PRE), post-exercise (POST), and $1 \mathrm{hr}$ recovery (RECOV). Sodium heparinized whole blood is used for cell counts, flow cytometry, and LPS stimulation. Serum is separated and stored at $-80^{\circ} \mathrm{C}$ for batch analyses of inflammatory cytokines and acute phase proteins, and biomarkers of metabolism. The main planned variables to be studied in this project are circulating classical (CD14+CD16-) and pro-inflammatory (CD14+CD16+) monocyte percentages, monocyte function, and T-cell subsets.

Heart rate variability

Participants are fitted with a Polar heart rate monitor (Polar V800) and then lay down for five minutes. After this five-minute rest, data is collected for ten minutes while the participant is laying supine. HRV is 
collected at visit one and after each exercise bout. The post-exercise bout HRV measurement is collected approximately 30 minutes after the end of exercise. R-R intervals will be analyzed with kubios HRV Analysis Software v.1.1 (Biomedical Signal Analysis Group, University of Kuopio, Finland).

\section{Recruitment}

Recruitment will be targeted based on the two age groups and physical activity status. Flyers and recruitment tables will be set up at locations to target physically active participants (fitness facilities, fitness clubs, sports clubs, etc.) and physically inactive participants (general health fairs). The older adult group will also be recruited from local senior centers, senior health fairs, and adult education classes. The young adult group will also be recruited from the student body at the University of Houston. Enrollment is anticipated to take two years.

Discontinuing or modifying the protocol

Participation is voluntary, and at any time the participant may request to not continue the study at no consequence to them. The participant may be removed from the study or data analyses upon reporting of new information that changes their responses to the inclusion/exclusion criteria (i.e. reporting of new medication or change in health status, etc.). If the participant is unable to complete the exercise bout at the prescribed intensity, the intensity may be reduced $2-5 \%$ so that the participant can complete the bout. Modifications outside this plan will be reported to the institutional review board.

\section{Statistical analyses}

Data analyses plan

For each outcome, we will fit a mixed effects model on the three factors and their interactions. Baseline values will act as a covariate, and the intercept in the change-from-baseline model will be tested to assess post-exercise effects within each physical activity status group and mode of exercise. Prior to model fitting, data will be transformed to improve model fit (i.e., normality, constant within-group variance). As only two time points will be included in the model, no adjustment for autocovariance will be possible. Contrasts will be used to compare individual timepoints across the three factors. Since testing will be done acutely, we assume that data missingness will be rare, largely due to problems with measurement or sample collection, and thus ignorable. Statistical significance will be declared for $\mathrm{p}$ values less than 0.05. Tukey Honest Significant Difference testing will be used to adjust alpha levels to control the Type I error rate. Covariates such as sex will be included in the model if they improve model fit 
without overfitting the data. If enough p-values fall below the Bonferroni-adjusted level to achieve no more than a 0.05 error rate, results may be reported with only adjusted $p$-values.

Sample size and power calculation

Sample size calculations were carried out based on expected differences within inflammatory monocytes (delta $=4.4, S D=3.7$ ), our primary variable. Values related to variability were suggested by previous studies on these same outcomes(2,10).

Randomization

To ensure equal distribution of CRE or RE bout first, eight independently generated randomization schedules were generated by the project biostatistician for the four groups (young physically active, young physically inactive, older physically active, older physically inactive, each stratified by sex). The biostatistician is not involved with recruitment or enrollment, and has no contact with the participants. A sequentially numbered list was kept, and as a participant is enrolled in the study and meets all study inclusion and exclusion criteria they are given the exercise bout assignment in order on the list. The co-investigator who consented the participant will assign the intervention, and there is no blinding.

Data storage and monitoring

Data entry will be performed by a member of the research team. A second member of the team will spot-check the data entry. Range checks will be conducted on every variable prior to statistical analyses. Biochemical assays will be performed in duplicate. All data is referenced by participant code. Data is stored on a password-protected university computer and server. Hard copies of data are stored in a locked cabinet in the Laboratory of Integrated Physiology. The informed consent is the only document with the participant's name, and is stored in a separate cabinet. De-identified datasets may be uploaded to public databases and/or available by request to the PI. Scientific manuscripts will be used to disseminate the study results. Participants may request a copy of their DEXA results for personal informational use.

A data monitoring committee was not formed for this study. The exercise interventions in this protocol are well-established to be safe and recommended ("standard of care") for the study population. The novelty of the protocol is in the study outcome variables, not the intervention. Any adverse event will be reported to the Institutional Review Board at the University of Houston. The investigators do not plan any formal audit, but the sponsor may randomly audit the study. 


\section{Discussion}

This is the first randomized trial to address potential differences in immune cell responses to two different modes acute exercise when including the age and physical activity status of the participants. These exercise responses need to be clarified, because aging can cause physiological changes that impact the immune system and the acute exercise response(11). Furthermore, there is often an ageassociated decline in physical activity that can exacerbate age-related changes in the immune system(12, 13). There is still uncertainty over how remaining physically active can "preserve" the aging immune system.

We anticipate that activity status (physically active or inactive) will have a bigger influence on the study variables than age. This is based on results from our previous studies where physically inactive older adults had a higher percentage of circulating pro-inflammatory CD14+CD16+ monocytes than physically active older adults $(2,3)$. Similarly, older adults with lower cardiorespiratory fitness levels had higher proportions of senescent T-cells than their fit counterparts(6). However, these previous studies did not include younger adult comparison groups, and/or multiple modes of exercise. Pro-inflammatory CD14+CD16+ monocytes are significant because in addition to producing large quantities of proinflammatory cytokines and very few anti-inflammatory cytokines(1), CD14+CD16+ monocytes are linked to atherosclerosis and contribute to the progression of atherosclerosis $(4,14)$. An accumulation of senescent, or highly-differentiated, T-cells is significant because these cells have high inflammatory properties, poor proliferative responses, and are often indicative of a reduction in the T-cell repertoire(5). Better comprehension of the acute exercise response could lead to a better understating of how exercise promotes healthy aging, and the role of the immune system health in healthy aging.

Despite the many strengths of this study, there are several limitations as well. Many of these limitations are due to the limitations of research in humans, and are based on assumptions that must be made when using human participants in research studies. For example, we are not controlling the diets of the research participants and assume that their diets are relatively stable between visits. We also must rely on the self-reported data to be accurate. The self-reported physical activity data, which is an important part of our study design, is being confirmed with accelerometers. Another limitation is that all participants are healthy. We are excluding individuals with known chronic disease (besides controlled hypertension), and this limits the relevance of the results. A follow-up study should focus on participants with a wider range of health histories.

Findings in this study will be useful for helping determine how exercise affects the immune system in people who meet the ACSM physical activity recommendations or do not. This will strengthen the rationale for the benefits of exercise as we age. It may also support potential mechanisms for how exercise prevents age-related chronic diseases in older adults who are physically active. Together, these results will add continued support towards encouraging individuals to maintain their physical activity throughout the lifespan. 


\section{Trial status}

At the time of submission, subject recruitment and data collection is on-going. Registered on clinicaltrials.gov January 4, 2019. Protocol version 20191211. Anticipated recruitment period January 4, 2019 - December 31, 2020.

\section{List Of Abbreviations}

ACSM American College of Sports Medicine

BMI Body mass index

CAARE Comparing Acute vs. Aerobic Resistance Exercise

CRE Cardiorespiratory exercise

DEXA Dual energy $x$-ray absorptiometry

HRV Heart rate variability

PANAS Positive and Negative Affect Schedule

PMSQ Profile of Mood States Questionnaire

POST Post-exercise

PRE Pre-exercise

RE Resistance exercise

RECOV Recovery

\section{Declarations}

Ethics approval and consent to participate

Written informed consent will be obtained from all research participants.

Acknowledgements

None to report at this time. 
Authors' contributions

MMM and RMG conceived the research design and study hypothesis. $\mathrm{KJ}$ developed the statistical analyses plan. MMM, RMG, and $\mathrm{KJ}$ contributed to writing and revising the manuscript, and approve the submission. Future manuscripts may include additional study team members who meet authorship guidelines, as described by

\section{Funding}

This project is funded by an internal award to Markofski by the University of Houston. The content is solely the responsibility of the authors and does not necessarily represent the official views of the University of Houston. The sponsor did not play a role in the study design, data collection and analysis, decision to publish, or preparation of the manuscript.

Availability of data and material

Data sharing is not applicable to this article as no datasets were generated or analyzed.

Ethics approval and consent to participate

This study was approved by the Institutional Review Board at the University of Houston (STUDY00001191).

Consent for publication

NA

Competing interests

The authors have no competing interests to report.

Author details

Melissa M. Markofski ${ }^{1}$, Rachel Graff ${ }^{1}$, and Kristofer Jennings ${ }^{2}$ 
${ }^{1}$ Department of Health and Human Performance, University of Houston, 3875 Holman St., Houston, TX 77204, USA.

${ }^{2}$ Department of Biostatistics, University of Texas M.D. Anderson Cancer Center, 1515 Holcombe Blvd., Houston, TX 77030, USA.

\section{References}

1. Belge KU, Dayyani F, Horelt A, Siedlar M, Frankenberger M, Frankenberger B, et al. The proinflammatory CD14+CD16+DR++ monocytes are a major source of TNF. J Immunol. 2002;168(7):3536-42.

2. Markofski MM, Flynn MG, Carrillo AE, Armstrong CL, Campbell WW, Sedlock DA. Resistance exercise training-induced decrease in circulating inflammatory CD14+CD16+ monocyte percentage without weight loss in older adults. Eur J Appl Physiol. 2014;114(8):1737-48.

3. Timmerman KL, Flynn MG, Coen PM, Markofski MM, Pence BD. Exercise training-induced lowering of inflammatory (CD14+CD16+) monocytes: a role in the anti-inflammatory influence of exercise? J Leukocyte Biol. 2008;84(5):1271-8.

4. Schlitt A, Heine G, Blankenberg S, Espinola-Klein C, Dopheide J, Bickel C, et al. CD14+CD16+ monocytes in coronary artery disease and their relationship to serum TNF-alpha levels. Thromb Haemost. 2004;92(2):419-24.

5. Brunner S, Herndler-Brandstetter D, Weinberger B, Grubeck-Loebenstein B. Persistent viral infections and immune aging. Ageing Res Rev. 2011;10(3):362-9.

6. Spielmann G, McFarlin BK, O'Connor DP, Smith PJ, Pircher H, Simpson RJ. Aerobic fitness is associated with lower proportions of senescent blood T-cells in man. Brain Behav Immun. 2011;25(8):1521-9.

7. Steppich B, Dayyani F, Gruber R, Lorenz R, Mack M, Ziegler-Heitbrock HW. Selective mobilization of CD14(+)CD16(+) monocytes by exercise. Am J Physiol Cell Physiol. 2000;279(3):C578-86.

8. American College of Sports Medicine, Riebe D, Ehrman JK, Liguori G, Magal M. ACSM's guidelines for exercise testing and prescription. Tenth edition. ed. Philadelphia: Wolters Kluwer; 2018. xxx, 472 pages $p$.

9. Epley B. Poundage Chart. Lincoln, NE: Body Enterprises; 1985.

10. Timmerman K, Flynn M, Coen P, Markofski M, Pence B. Exercise training-induced lowering of inflammatory (CD14+CD16+) monocytes: a role in the anti-inflammatory influence of exercise? J Leukoc Biol. 2008;84(5):1271-8.

11. Ferraro KF, Wilmoth JM. Gerontology perspectives and issues. New York, NY: Springer; 2013.

12. Minuzzi LG, Rama L, Bishop NC, Rosado F, Martinho A, Paiva A, et al. Lifelong training improves antiinflammatory environment and maintains the number of regulatory $T$ cells in masters athletes. Eur $\mathrm{J}$ Appl Physiol. 2017;117(6):1131-40. 
13. Silva LC, de Araujo AL, Fernandes JR, Matias Mde S, Silva PR, Duarte AJ, et al. Moderate and intense exercise lifestyles attenuate the effects of aging on telomere length and the survival and composition of T cell subpopulations. Age. 2016;38(1):24.

14. Angelovich TA, Hearps AC, Jaworowski A. Inflammation-induced foam cell formation in chronic inflammatory disease. Immunol Cell Biol. 2015;93(8):683-93.

\section{Figures}

\begin{tabular}{|c|c|c|c|c|c|}
\hline & \multicolumn{5}{|c|}{ STUDY PERIOD } \\
\hline \multirow[b]{2}{*}{ TIMEPOINT } & Enrolment & Allocation & \multicolumn{3}{|c|}{ Post-allocation } \\
\hline & -7 to $-14 d$ & 0 & $P R E$ & POST & RECOV \\
\hline \multirow{3}{*}{$\begin{array}{l}\text { Eligibility screen } \\
\text { Informed consent }\end{array}$} & $\mathrm{X}$ & & & & \\
\hline & $\mathrm{X}$ & & & & \\
\hline & & $\mathrm{X}$ & & & \\
\hline \multicolumn{6}{|l|}{$\begin{array}{l}\text { Cardiorespiratory } \\
\text { exercise session }\end{array}$} \\
\hline \multicolumn{6}{|l|}{$\begin{array}{r}\text { Resistance } \\
\text { exercise session }\end{array}$} \\
\hline \multirow[t]{2}{*}{$\begin{array}{r}D E X A \text { and fitness } \\
\text { tests }\end{array}$} & $\mathrm{X}$ & & & & \\
\hline & & & $\mathrm{X}$ & $x$ & $x$ \\
\hline \multirow{2}{*}{$\begin{array}{l}\text { Heart rate } \\
\text { variability }\end{array}$} & & & $\mathrm{X}$ & & $\mathrm{x}$ \\
\hline & & & $\mathrm{X}$ & $\mathrm{X}$ & $\mathrm{X}$ \\
\hline
\end{tabular}

Figure 1

Schedule of enrollment, allocation, and data collection for CAARE protocol.

\section{Supplementary Files}

This is a list of supplementary files associated with this preprint. Click to download.

- SPIRITChecklistforrandomisedstudies.doc 